\title{
Study on the expression of MMP-9 and NF-KB proteins in epithelial ovarian cancer tissue and their clinical value
}

\author{
Shen Wei ${ }^{1, a}$, Chen Juan ${ }^{2}$, Li Xiurong ${ }^{1}$ and Yin Jie ${ }^{1}$ \\ ${ }^{1}$ Department of Obstetrics and Gynecology, the Forth Hospital of Hebei Medical University, Shijiazhuang, \\ 050011, Hebei, China \\ ${ }^{2}$ Health Examination Center, the Fourth Hospital of Hebei Medical University, Shijiazhuang, 050011, Hebei, \\ China
}

\begin{abstract}
Objective: To investigate the expression of matrix metalloproteinase-9 (MMP-9) and nuclear factor карpa-B $(\mathrm{NF}-\kappa \mathrm{B})$ in different ovarian tissue and explore the relationship between their expression and clinicopathological features of epithelial ovarian cancer. Methods: The expression of NF- $\mathrm{KB}$ and MMP-9 in 15 cases of normal ovarian tissue and 80 cases of epithelial ovarian cancer were detected by mmunohistochemistry PV method. Results: Expression of MMP-9 and NF- $\mathrm{BB}$ in epithelial ovarian cancer was significantly higher than those in normal ovarian, and the difference was statistically significant $(P<0.05)$. The expression of MMP-9 protein was not related to histology classification and differentiation degree, but it was significantly related with lymphatic metastasis and clinical stage $(P<0.05)$. The expression of NF- $\kappa \mathrm{B}$ protein was significantly associated with histology classification, lymphatic metastasis, clinical stage, and differentiation degree $(P<0.05)$. In epithelial ovarian cancer tissue, the expression of MMP-9 and NF- $\kappa B$ proteins showed a significant positive correlation $(P<0.01)$. Conclusion: The expression MMP-9 and NF- $\kappa \mathrm{B}$ proteins are closely related to pathological features of epithelial ovarian cancer and there are important significance to the development, invasion and metastasis of epithelial ovarian cancer. So they might become useful prognostic indicator for diagnosis and prognosis of epithelial ovarian cancer.
\end{abstract}

\section{Introduction}

Ovarian cancer was one of the common malignant tumors for women. Its incidence rate ranked third next to the cervical cancer and uterine cancer, but its mortality rate was of being first on the above three kinds of malignant tumors of female genital tract. Its occurrence and development was a complicated process involved in many ways and many genes [1]. Matrix metalloproteinase-9 (MMP9), a key enzyme involved in the degradation of extracellular matrix, was closely related to tumor invasion and metastasis [2]. Nuclear factor kappa B $(\mathrm{NF}-\kappa \mathrm{B})$ was a eukaryotic transcription factor, which existed in almost all cells. NF- $\mathrm{BB}$ was usually the form of homologous or heterologous dimer, and its most common form was P50/P65 dimer, which played a passing information role in the process of cell differentiation, apoptosis and tumor growth [3]. In this study, the expression of MMP-9 and NF- $\kappa \mathrm{B}$ proteins in normal ovarian tissue and epithelial ovarian carcinoma was detected by immunohistochemical method. The relationship between MMP-9 and NF- $\mathrm{BB}$ protein expression was

\footnotetext{
${ }^{\mathrm{a}}$ Corresponding author: shenwei1980112@163.com
} 
analyzed, in order to provide valuable theoretical basis for the mechanism of invasion and metastasis of ovarian cancer and prognosis effect.

\section{Material and method}

\subsection{General data}

95 cases of epithelial ovarian cancer specimens, which were from ovarian cancer tissue of gynecologic surgical resection, were collected from the fourth hospital of Hebei Medical University during January 2010-January 2012 year. A total of 80 patients with epithelial ovarian cancer were selected, whose age were from 43 to 75 years, with a median age of 55.8 years, including 41 cases of serous cystadenocarcinoma, 28 cases of mucinous cystadenocarcinoma, and 11 cases of endometrioid adenocarcinoma. Histological staging was performed according to NCCN2012. In 80 cases of specimens, there were 38 cases in stage I and II, 42 cases in stage III and IV, 39 cases of welldifferentiated, 26 cases of moderately differentiated, and 15 cases of poorly differentiated, 39 cases with lymphatic metastasis, and 41 cases without lymphatic metastasis. 15 cases of normal ovarian tissue were collected, which were obtained by double adnexectomy for uterine myoma. The median age was from 36 to 58 years, and the median age was 42.3 years. All the specimens were confirmed by pathological examination, and the data were complete. All patients had not received chemotherapy or radiotherapy before operation.

\subsection{Main reagents}

Rabbit anti human NF- kappa b/P65 polyclonal antibody and Rabbit anti human MMP-9 polyclonal antibody were purchased from Santa Cruz Company. PV-6001 and PV-6002 detection reagents and DAB kit were purchased from Beijing Zhongshan-jinqiao Reagent Company. PBS buffer solution was purchased from GIBCO Company.

\subsection{Immunohistochemical staining method}

Immunohistochemistry PV method was used in experiment. Surgical specimens were fixed in $10 \%$ formalin, embedded in paraffin. Paraffin specimens were separated into $5 \mathrm{~mm}$ sections. After conventional dewaxing, they were immersed in $3 \%$ hydrogen peroxide methanol solution at room temperature for 10-15 min to eliminate endogenous peroxidase activity. After the antigen restore used autoclave for $2 \mathrm{~min}$, they were sealed with $1 \%$ BSA (bovine serum albumin), incubated at room temperature for 10-15 min (closed tissue charge), and poured to serum. Adding primary antibody NF$x \mathrm{~b} / \mathrm{P} 65$ and MMP-9 (titer 1: 50), they were placed in a refrigerator at $4{ }^{\circ} \mathrm{C}$ for the night, washed with $0.01 \mathrm{M}$ PBS, and incubated for 5 minute for 3 times, and then adding the secondary antibody PV-6001 and PV-6002 reagent, they were incubated at $37^{\circ} \mathrm{C}$ for $60 \mathrm{~min}$. They were vibrated wash in $0.01 \mathrm{M}$ PBS for $5 \mathrm{~min}$ for 3 times, and stained (PBS $4 \mathrm{ml}$, DAB $5 \mathrm{~g}, \mathrm{H}_{2} \mathrm{O} 25 \mathrm{ul}$ ) around 5 min (with a microscopically moderate as well). They were washed 5 times by the distilled water, redyed with hematoxylin for $1 \mathrm{~min}$, dehydrated, hyalinized with xylene, sealed with neutral gum, and observed under the light microscope.

\subsection{Result determination}

MMP-9 and NF- $x \mathrm{~B}$ were detected positive, if brown-yellow granules were discovered in cytoplasm. Comprehensive evaluation methods: The score of the degree of staining was as follows: Colourless for 0 , light yellow for 1 , claybank for 2 , brown for 3 . The counting of the ratio of positive cell, which was positive cells accounted for the percentage of tumour cells, was as follows: less or equal to $5 \%$ for 0 , $6-25 \%$ for $1,26-50 \%$ for $2,>50 \%$ for 3 . Assessment: Multiplication of the above two score value 
was adopted, and the result was 0 for the negative (-), 1- 2 for weak positive (+), 3- 4 for moderate positive $(++)$, and $>4$ for strong positive $(+++)$.

\subsection{Statistical methods}

The SPSS13.0 statistical software was used, and measuring data was expressed by $(\bar{x} \pm s)$. The correlation among the positive rates was analyzed by $\chi^{2}$ test. The correlation analysis of protein expression was analyzed by Spearman correlation analysis. $P<0.05$ was statistically significant.

\section{Results}

\subsection{The expression of MMP-9 and NF-KB proteins in different ovarian tissue}

The positive expression rate of MMP-9 and NF- $x \mathrm{~B}$ proteins in epithelial ovarian carcinoma tissue, was significantly higher than that in normal ovarian tissue, and the difference was statistically significant $(P<0.05$, Table 1$)$. The results of MMP-9 and NF- $x$ B proteins in epithelial ovarian carcinoma and normal ovarian tissue were detected by PV method, and the results were shown in Figure 1.

Table 1. Expression of MMP-9 and NF- $\varkappa \mathrm{B}$ proteins in different ovarian tissue

\begin{tabular}{|c|c|c|c|c|c|}
\hline \multirow{2}{*}{ Group } & \multirow{2}{*}{$\mathbf{n}$} & \multicolumn{2}{|c|}{ MMP-9 expression } & \multicolumn{2}{|c|}{ NF- $\kappa B$ expression } \\
\hline & & $\mathrm{n}$, positive rate $(\%)$ & P-value & $\mathrm{n}$, positive rate $(\%)$ & P-value \\
\hline Normal ovarian & 15 & $0(0)$ & \multirow{2}{*}{$<0.01$} & $1(6.7)$ & \multirow{2}{*}{$<0.01$} \\
\hline $\begin{array}{l}\text { Epithelial ovarian } \\
\text { carcinoma }\end{array}$ & 80 & $54(67.5)$ & & $62(77.5)$ & \\
\hline
\end{tabular}
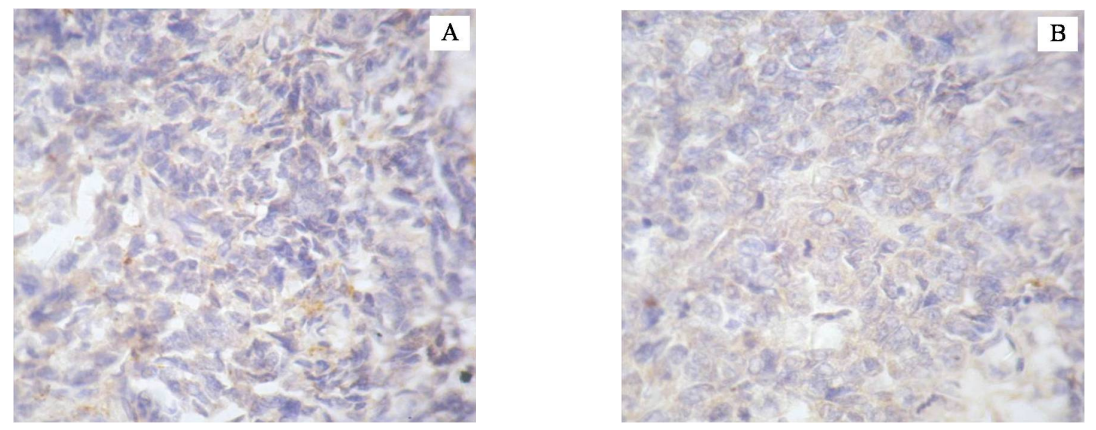

Figure 1. Immunohistochemical results of MMP-9 and NF- $\mathrm{BB}$ proteins in epithelial ovarian carcinoma. (A) Positive expression of MMP-9 protein; (B) Positive expression of NF- kB protein.

\subsection{Correlation between MMP-9 protein expression and clinicopathological parameters in epithelial ovarian cancer tissue}

The positive expression rate of MMP-9 protein among serous cystadenocarcinoma, mucinous cystadenocarcinoma and endometrioid adenocarcinoma had no significant difference $(P>0.05)$. The positive expression rate of ovarian cancer tissue in stage I - II was lower than that of ovarian cancer in stage III - IV, and the difference was statistically significant $(P<0.01)$. In ovarian cancer tissue of the 
high differentiation, middle differentiation and low differentiation, the positive expression rate gradually increased with the decrease of the differentiation degree, but there was no significant difference $(P>0.05)$. In ovarian cancer tissue, the positive expression rate of ovarian cancer tissue with lymphatic metastasis was higher than that without metastasis $(P<0.05$, Table 2$)$.

\subsection{Correlation between NF-kB protein expression and clinicopathological parameters in epithelial ovarian cancer tissue}

The positive expression rate of $\mathrm{NF}-\varkappa \mathrm{B}$ protein in serous cystadenocarcinoma, mucinous cystadenocarcinoma and endometrioid adenocarcinoma gradually increased, and there was significant difference $(P<0.05)$. The positive expression rate of stage I - II of ovarian cancer tissue was lower than that of stage III - IV, the difference was statistically significant $(P<0.01)$. With the reduced of differentiation, the positive expression rate of ovarian cancer tissue significantly increased, and the difference was statistically significant $(P<0.05)$. The positive expression rate of ovarian cancer tissue with lymphatic metastasis was higher than that without metastasis, and the difference was statistically significant $(P<0.01$, Table 2$)$.

Table 2. Expression of MMP-9 and NF- $\varkappa \mathrm{B}$ proteins and their correlation with clinicpathological features of epithelial ovarian cancer

\begin{tabular}{|c|c|c|c|c|c|}
\hline \multirow{2}{*}{ Characteristics } & \multirow{2}{*}{$\mathbf{n}$} & \multicolumn{2}{|c|}{ MMP-9 expression } & \multicolumn{2}{|c|}{ NF- $\kappa B$ expression } \\
\hline & & $\mathrm{n}$, positive rate $(\%)$ & P-value & n, positive rate $(\%)$ & P-value \\
\hline \multicolumn{6}{|l|}{ Histology classification } \\
\hline Serous cystadenocarcinoma & 41 & $26(63.4)$ & \multirow{3}{*}{$>0.05$} & $27(65.9)$ & \multirow{3}{*}{$<0.05$} \\
\hline Mucinous cystadenocarcinoma & 28 & $20(71.4)$ & & $24(85.7)$ & \\
\hline Endometrioid adenocarcinoma & 11 & $8(72.7)$ & & 11(100) & \\
\hline \multicolumn{6}{|l|}{ Clinical stage } \\
\hline I-II & 38 & $18(47.4)$ & \multirow{2}{*}{$<0.01$} & $22(57.9)$ & \multirow{2}{*}{$<0.01$} \\
\hline III-IV & 42 & $36(85.7)$ & & $40(95.2)$ & \\
\hline \multicolumn{6}{|l|}{ Differentiation degree } \\
\hline High & 39 & $25(64.1)$ & \multirow{3}{*}{$>0.05$} & $25(64.1)$ & \multirow{3}{*}{$<0.05$} \\
\hline Middle & 26 & $18(69.2)$ & & $23(88.5)$ & \\
\hline Low & 15 & $11(73.3)$ & & $14(93.3)$ & \\
\hline \multicolumn{6}{|l|}{ Lymphatic metastasis } \\
\hline Positive & 39 & $32(82.1)$ & \multirow{2}{*}{$<0.05$} & $36(92.3)$ & \multirow{2}{*}{$<0.01$} \\
\hline Negative & 41 & $22(53.7)$ & & $26(63.4)$ & \\
\hline
\end{tabular}




\subsection{The relationship between MMP-9 and NF- KB protein expression in epithelial ovarian cancer}

The analysis of 80 cases of samples of ovarian cancer tissue by Spearman rank correlation showed that the MMP-9 protein expression was positively correlated with NF- $x$ B protein expression in ovarian cancer tissue $(P<0.01)$.

\section{Discussions}

The formation and development of tumor were related to the activation of oncogenes or gene inactivation of antioncogene for cell gene mutation, and they were also influenced by the changes of the microenvironment of the tumor cells. Matrix metalloproteinases (MMPs) were a class of proteins with very close relationship with the invasion and metastasis of tumor. MMP-9 was an important member of the MMPs family, also known as type IV collagenase and gelatinase B, and could degrade the main components in the cell membrane matrix, namely type IV and V collagen and gelatin, which resulted in the tumor cell invasion to the surrounding tissue through the basement membrane loss, thereby promoted tumor invasion and metastasis. In recent years, the studies found that MMP-9 played an important role in the evolution of malignant tumor. In thyroid papillary carcinoma, nonsmall cell lung cancer, primary carcinoma of liver and other tissue, MMP-9 showed high expression, and was closely related with tumor progression. It was a sensitive biological indicator by used as preoperative diagnosis, judging postoperative prognosis and determining with and without lymph node metastasis [4-5]. This paper studies found that MMP-9 protein was high expression in ovarian tumor tissue, and the positive expression rate was closely related to clinical staging and lymph node metastasis. It suggested that the expression of MMP-9 was involved in the invasion and metastasis of ovarian cancer, so it could be used as one of the judgment index of prognosis of ovarian cancer patients.

$\mathrm{NF}-x \mathrm{~B}$ was a nuclear protein found in $\mathrm{B}$ cells that binded to the $\mathrm{B}$ site of the immunoglobulin kappa light chain enhancer and could regulate the transcription of the immunoglobulin kappa light chain, so it was named nuclear transcription factor $\varkappa \mathrm{B}$. NF $-\varkappa \mathrm{B}$, as a kind multidirectional and multifunctional nuclear transcription factor, could inhibit apoptosis by inducing the expression of antiapoptotic genes. It had a close relationship with occurrence and development of tumor and chemotherapy drug resistance, and acted a key role in the anti apoptosis mechanism of tumor. Zhang Jian et al by transfection of $\mathrm{I} \varkappa \mathrm{B} \alpha$ gene demonstrated that NF- $\varkappa \mathrm{B}$ activation in A549 cells could inhibit their invasion ability, and abnormal expression and activation of NF- $x \mathrm{~B}$ was also detected in esophageal squamous cell carcinoma, gastric carcinoma, liver cancer and other tissue [6-8]. This paper studies showed that NF- $x \mathrm{~B}$ in ovarian cancer tissue was significantly higher than that in normal ovarian tissue, and was significantly correlated with histological type, lymph node metastasis, clinical stage, degree of differentiation, further suggesting that the expression of NF- $\varkappa \mathrm{B}$ protein up-regulation could be an important mechanism of the occurrence and development of ovarian cancer.

Kim $\mathrm{Y}$ et al found that MMP-9 gene promoter region contained multiple binding sites of NF- $x \mathrm{~B}$ transcription factor, and inhibition of NF- $x \mathrm{~B}$ activity could significantly inhibit the expression of MMP-9 gene [9]. Shishodia $S$ et al found that NF- $\chi B$ could promote MMP-9 expression in lung cancer, and they in cervical cancer, thyroid papillary cancer, lung cancer were positively correlated [10]. The above results showed that MMP-9 and NF- $x \mathrm{~B}$ protein expression in epithelial ovarian cancer was positively correlated, and $\mathrm{NF}-\varkappa \mathrm{B}$ activation could regulate the transcription and expression of MMP-9 in ovarian cancer cells, and promote the invasion and migration of ovarian cancer cells.

\section{References}

1. A. Jemal, R. Siegel, E. Ward, J. Xu, M.J. Thun, CA-Cancer J. Clin., 58, 71(2008)

2. L. Liu, J. Wu, Z. Ying, M. Li, Cancer Res., 70, 3750(2010)

3. J. Liu, G. Yang, Cancer Res., 64, 1655(2004) 
4. K. Zarrabi, A. Dufour, J. Li, J. Cao, J. Biol. Chem., 286, 33167(2011)

5. Y. Koyama, H. Naruo, Y. Yoshitomi, K. Oguri, J. Biochem., 143, 581(2008)

6. J. Zhang, Y.J. Xu, W.N. Xiong, Z.X. Zhang, C.L. Du, L.F. Qiao, W. Ni, S.X. Chen, Chin. J. Cancer, 27, 710(2008)

7. B.B. Aggarwal, Cancer Cell, 6, 203(2004)

8. A.C. Bharti, B.B. Aggarwal, Biochem. Pharmacol., 64, 883(2002)

9. Y. Kim, H. Kang, S.W. Jang, J. Ko, Cell Physiol. Biochem., 28, 175(2011)

10. S. Shishodia, P. Potdar, C.G. Gairola, B.B. Aggarwal, Carcinogenesis, 24, 1269(2003) 